\title{
Drug Used Since Last Visit
}

National Cancer Institute

\section{Source}

National Cancer Institute. Drug Used Since Last Visit. NCI Thesaurus. Code C157392.

An indication that a specified drug was used during the time since the last visit. 